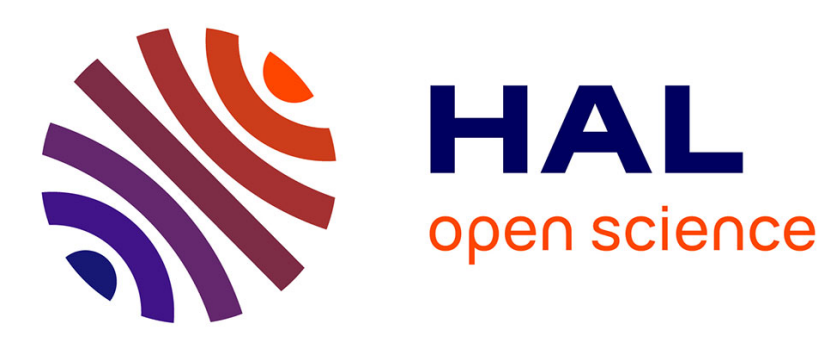

\title{
An energy-efficient leader election mechanism for wireless body area networks
}

Rongrong Zhang, Hassine Moungla, Ahmed Mehaoua

\section{To cite this version:}

Rongrong Zhang, Hassine Moungla, Ahmed Mehaoua. An energy-efficient leader election mechanism for wireless body area networks. IEEE Global Communications Conference 2014, Dec 2014, Austin, United States. pp.2411 - 2416 10.1109/GLOCOM.2014.7037169 . hal-01270445

\section{HAL Id: hal-01270445 \\ https://hal.science/hal-01270445}

Submitted on 17 Feb 2016

HAL is a multi-disciplinary open access archive for the deposit and dissemination of scientific research documents, whether they are published or not. The documents may come from teaching and research institutions in France or abroad, or from public or private research centers.
L'archive ouverte pluridisciplinaire HAL, est destinée au dépôt et à la diffusion de documents scientifiques de niveau recherche, publiés ou non, émanant des établissements d'enseignement et de recherche français ou étrangers, des laboratoires publics ou privés. 


\title{
An Energy-Efficient Leader Election Mechanism for Wireless Body Area Networks
}

\author{
Rongrong Zhang*, Hassine Moungla*, Ahmed Mehaoua* \\ ${ }^{*}$ LIPADE, University of Paris Descartes, Sorbonne Paris Cité 45 rue des saints pères, 75006, Paris, France \\ Email: \{rongrong.zhang; hassine.moungla; ahmed.mehaoua $\} @$ parisdescartes.fr
}

\begin{abstract}
In Wireless Body Area Networks (WBANs), the energy consumption determines the lifetime of the entire network. As a result, how to conserve the energy to prolong the network lifetime becomes a key problem in WBANs. In this paper, to address the energy conservation problem in WBANs, we develop an Energy-Efficient Leader Election mechanism, called EELE. In EELE, each node competes for the leader following the distributed leader election algorithm in which a utility function is constructed with the consideration of the residual energy and the location of the node. Moreover, a distance-aware hybrid communication mode is proposed such that a node can choose either direct communication or cooperative communication to alleviate the burden of the leader or the far node. Extensive simulation results demonstrate the effectiveness and the efficiency of EELE mechanism in terms of longer network lifetime, better energy characteristics and higher throughput.
\end{abstract}

\section{INTRODUCTION}

Wireless Body Area Networks (WBANs) are the emerging networks designed and developed for human body to monitor, manage and communicate the real time physiological parameters such as the temperature, blood pressure, heart rate, electrocardiogram (ECG), and electroencephalogram (EEG) [1]. Due to the highly extensive potential applications, for instance, medical treatment and diagnosis, public safety and preventing medical accidents, etc [2], WBANs have been paid great attention. A WBAN typically consists of a collection of lower-power, miniaturised, lightweight devices with sensor capabilities on, around or implanted in the human body [3] The main two types of devices can be distinguished: sensor and Personnel Digital Assistant (PDA) which acts as a sink The sensors are used to measure certain parameters of the human body, either externally or internally, while the main purpose of the sink is to collect all the information attained by the sensors and communicate it to the user (patient, nurse, etc.) via an external gateway.

On account of the low power level and scarce battery capability, a fundamental problem in WBANs is to maximize the network lifetime. In general, network longevity can be achieved by energy conservation [4]. To reduce energy consumption, a number of energy efficient protocols for Wireless Sensor Networks (WSNs) have been proposed, among which sleep mechanism and clustering mechanism extensively attract more attention [5-9]. Sleep mechanism alternates each node between active and sleep status by turning the radio on and off periodically to save energy substantially, while cluster scheme partitions the network into a set of clusters with a cluster head and some ordinary nodes as its members.

Heinzelamn et. al. [6] study a Low-Energy Adaptive Cluster Hierarchy (LEACH) protocol for wireless microsensor networks. In LEACH, a node has a certain probability of becoming a cluster head, and the task of being a cluster head is rotated between nodes to balance the energy consumption and to avoid some nodes die earlier. The authors propose an enhancement of low energy adaptive clustering by introducing a new parameter for electing cluster head in [7]. Nodes with the highest remaining energy and the lowest energy variance consumption become the cluster heads with high probability. The additional variance parameter takes into account energy consumption dispersion if the considered node is elected as a cluster head. The dispersion highly depends on the relative positioning of the node to the base station.

Besides, a Hybrid Energy-Efficient Distributed Cluster (HEED) approach is proposed for ad hoc sensor networks in [8] where the cluster head is elected according to the residual energy. In the implementation of HEED, multi-hop is used when cluster heads deliver the data to the sink. In [9], the author proposes and evaluates two clustering-based protocols for heterogeneous WSNs, which are called Singlehop Energy-Efficient Clustering Protocol (S-EECP) and Multihop Energy-Efficient Clustering Protocol (M-EECP). In SEECP the cluster heads are elected by a weighted probability based on the ratio between residual energy of each node and average energy of the network. The nodes with high initial energy and residual energy will have more chance to be elected as cluster heads than those with low energy. Whereas in MEECP, the elected cluster heads transfer the data to the base station via multi-hop communication approach.

However, due to the typical features of WBANs, such as the limited energy resources and special communication medium, the existing protocols for the large-scale WSNs are not exactly applicable to the WBANs. Therefore, it is challenging and necessary to design an energy-efficient mechanism with the consideration of the characteristics of WBANs.

Inspired by the exiting work, in this paper, we propose an Energy-Efficient Leader Election (EELE) mechanism for WBANs. Specifically, EELE uses a distributed leader election algorithm where the leader is elected by localized competition.

Our key contributions are as follows:

- We partition a WBAN into a number of regions to manage sensor nodes efficiently. Furthermore, A distance-aware hybrid communication approach is introduced which ben- 
efits to relieve the burden of the far nodes and the leader;

- We develop a distributed leader election algorithm with the characteristics of WBANs taken into account, where the competitive probability with relation to the monitoring function of the sensor node and the utility function considering the influence of the location and the residual energy of the sensor node are constructed. And the algorithm turns out to be of low overhead and to be applicable to WBANs;

- We comprehensively evaluate the proposed mechanism and compare it against existing solutions. Simulation results demonstrate the effectiveness and the efficiency of the mechanism in terms of network lifetime, energy characteristics and throughput which are defined later.

The rest of this paper is organized as follows. In Section II, we present the network model and the hybrid communication mode in WBANs. The proposed distributed leader election algorithm and EELE mechanism are described in Sections III and IV, respectively. In Section V, we evaluate the performance of our work. The work is concluded in Section VI.

\section{Preliminaries}

In this section, we first describe the network model based on the region partition and the energy consumption model in WBANs. Then, the hybrid communication mode is introduced with the consideration of distance.

\section{A. Network model}

In order to achieve high energy efficiency and enhance the network scalability, a WBAN will be partitioned into $M$ logical regions. We assume that there are $I$ nodes in each region, and they can be classified into $K$ types based on their separate monitoring functions. Moreover, to conveniently distinguish different nodes, each node is assigned a RID, a $T I D$ and a NID to denote the region number, the type number, and the node number, respectively. Furthermore, the $m$-th region is represented by $r_{m}$ and the corresponding region set $R=\left\{r_{1}, r_{2}, \ldots, r_{M}\right\}$, where $|R|=M$. Correspondingly, the $k$-th type node is indicated as $t_{k}$ and the type set $T=\left\{t_{1}, t_{2}, \ldots, t_{K}\right\}$, where $|T|=K$. Also, the $i$-th node is described as $n_{i}$ and the node set, $N=\left\{n_{1}, n_{2}, \ldots, n_{I}\right\}$, where $|N|=I$. Therefore, we can use the triple $<r_{m}, t_{k}, n_{i}>$ to indicate uniquely the $i$-th node of the $k$-th type in the $m$-th region. Note that in this paper, we will use the terms of "node" and "sensor node" interchangeably to refer to the same WBAN entity.

Besides, for analytical tractability, we make the following reasonable assumptions about the nodes in a WBAN:

- All nodes are stationary after the deployment. And they also know the relevant information about themselves, such as the location and the real-time residual energy;

- All nodes are of the same sensing and computation capability. And they always have packets to send during their active periods;

- The sink is energy unlimited and is located in the center of the body. And all nodes can transmit directly with enough power to reach the sink if needed.
In WBANs, most of the devices are attached on human body, so the communications between nodes and the sink occur along the surface of the human body which contributes attenuation to radio signal. As we are just interested in the energy consumption of the communication, which is much larger than the energy used for sensing, we ignore the later in this paper. According to the radio hardware energy dissipation model shown in [10], the energy consumption model for transmitting and receiving $l$-bits data over distance $d$ is:

$$
\begin{aligned}
E_{T X}(l, d, n) & =l E_{\text {Txelec }}+l \epsilon_{a m p} d^{n}, \\
E_{R X}(l) & =l E_{\text {Rxelec }},
\end{aligned}
$$

where $E_{\text {Txelec }}$ and $E_{R x e l e c}$ are the energy dissipated by the radio to run the circuitry for the transmitter and the receiver respectively, and $\epsilon_{a m p}$ is the energy for the transmission amplifier. $d$ is the distance between the transmitter and the receiver, whereas $n$ represents the path loss coefficient ( $n=3.11$ for the LOS (Line-Of-Signal) channel and $n=5.9$ for the NLOS (Non-Line-Of-Signal) channel). Particularly, if one node is elected as the final leader, it will aggregate the original packets from the normal nodes into one single length-fixed packet and then send it to the sink, so the leader will consume extra $E_{D A}$ energy for the data aggregation.

\section{B. Hybrid communication mode}

In wireless communications, nodes can adopt direct communication or cooperative communication. Under the direct communication, each node can send its data directly to the sink in WBANs. But if the node is far away from the sink, the direct communication will require a large amount of transmission power (as shown in Equation (1)), which will quickly drain the battery of the far node and shorten the network lifetime. Therefore, the cooperative communication is exploited, in which the packets from the far nodes can be relayed to the sink by an intermediate node, so that the energy dissipation of the far node will be reduced. However, the cooperative communication is not suitable for the nodes close to the sink. If a closer node also employs cooperative communication, it will not only consume its own energy, but also consume the energy of the intermediate node.

Motivated by the aforementioned analysis, we can draw a conclusion that the direct communication is more energyefficient for the closer node, while the cooperative communication is more energy-efficient for the farther node. Therefore, a hybrid communication mode which is more suitable for WBANs is developed as follows. In each region, there is only one intermediate node which is in the term of "leader" afterwards. Correspondingly, the other nodes will be in the term of "normal node". The leader will aggregate the packets from the normal nodes and transfer it to the sink in cooperative communication. For a normal node, it can choose the communication mode according to the distance $\left(D_{1}\right)$ between it and the sink and the distance $\left(D_{2}\right)$ between the leader and the sink. If $D_{1}>D_{2}$, it will choose cooperative communication mode. Otherwise, the direct communication mode will be selected.

Consequently, when direct communication mode is used the leader is relieved of its relaying burden for nodes closer to 
the sink. Similarly, when cooperative communication mode is used the nodes far from the sink are relieved of their burden of long range transmissions to the sink. Thus by adaptively adjusting communication mode it is probable to conserve energy consumption and obtain an uniform load distribution.

\section{LEADER Election Algorithm}

In this section, we develop a distributed leader election algorithm based on utility function for WBANs. Furthermore, the complexity analysis of this algorithm will be presented.

\section{A. Algorithm design}

Considering the WBANs characteristics, one tentative leader is randomly chosen from each type. Let $g_{k}$ define the number of the nodes falling into the $k$-th type, satisfying $\sum_{k=1}^{K} g_{k}=I$. If node $n_{i}$ is affiliated with the $k$-th type, it will compete to be a tentative leader with the probability $P_{i}(t)$ at time $t$,

$$
P_{i}(t)=1 / g_{k} \text {. }
$$

So the expected number of the tentative leader per round is $K$. Then the optimal one among them is elected to be the final leader according to the utility function. In order to balance the task of becoming as the leader, the utility function is related with the residual energy and the distance between the node and the sink. For node $n_{i}$ the utility function at time $t$ is:

$$
U F(i, t)=\left(\frac{R_{0}}{d(i, \sin k)}\right)^{\alpha} \cdot\left(\frac{R E(i, t)}{E_{0}}\right)^{1-\alpha},
$$

where $R_{0}$ is the region communication radius, and $d(i, \operatorname{sink})$ denotes the distance between node $n_{i}$ and the sink. $E_{0}$ and $R E(i, t)$ are the initial energy and the residual energy of node $n_{i}$ at time $t$, respectively. $\alpha \in[0,1]$ is the weight that indicates the preference for the distance or the residual energy. Particularly, when $\alpha=0$, it implies the residual energy is the only criterion to be the final leader. And when $\alpha=1$, it means that the node closest to the sink will be the final leader.

In the proposed algorithm, an arbitrary tentative leader $n_{i}$ maintains a set $C_{n i}$ of its competitors. A Competitor $n_{j}$ is also a tentative leader with the same RID as $n_{i}$ but different TID from $n_{i}$. Whether a tentative leader becomes as the final leader successfully depends on its utility function. If one node win as the leader, the other normal nodes will act as its members. A formal description of the disposed algorithm is shown in the Algorithm 1.

In the leader election algorithm, the broadcast radius of every control message is $R_{0}$, thus $n_{i}$ can receive all message from the nodes in its $C_{n i}$. Initially, several nodes are elected to be tentative leaders with the probability $P_{i}(t)$. And the other nodes keep sleeping until the final leader is selected. If one node becomes the tentative node successfully, it will broadcast a COMPETE_MSG message which contains its $R I D, T I D, N I D$, and $R E$ as line 5-7 in the algorithm 1. Then, if node $n_{i}$ receives a COMPETE_MSG from node $n_{j}$ with the same region number, it will add $n_{j}$ to its competitor set $C_{n i}$. After the competitor set has been formed in lines 8-11, each tentative leader checks its utility function and makes a decision whether it can act as the final leader as in lines 1221 . Once $n_{i}$ finds its utility function more than that of the other nodes in $C_{n i}$, it will win as the leader successfully and will broadcast a LEADER_MSG. In case of the same utility value, we choose the node with more $R E$. Otherwise, if node $n_{i}$ receives a LEADER_MSG from $n_{j}$ in lines $22-25$, it will give up the competition immediately, and inform other nodes by a QUIT_MSG. Or else, it will remove $n_{j}$ from its $C_{n i}$ once receiving a QUIT_MSG from $n_{j}$.

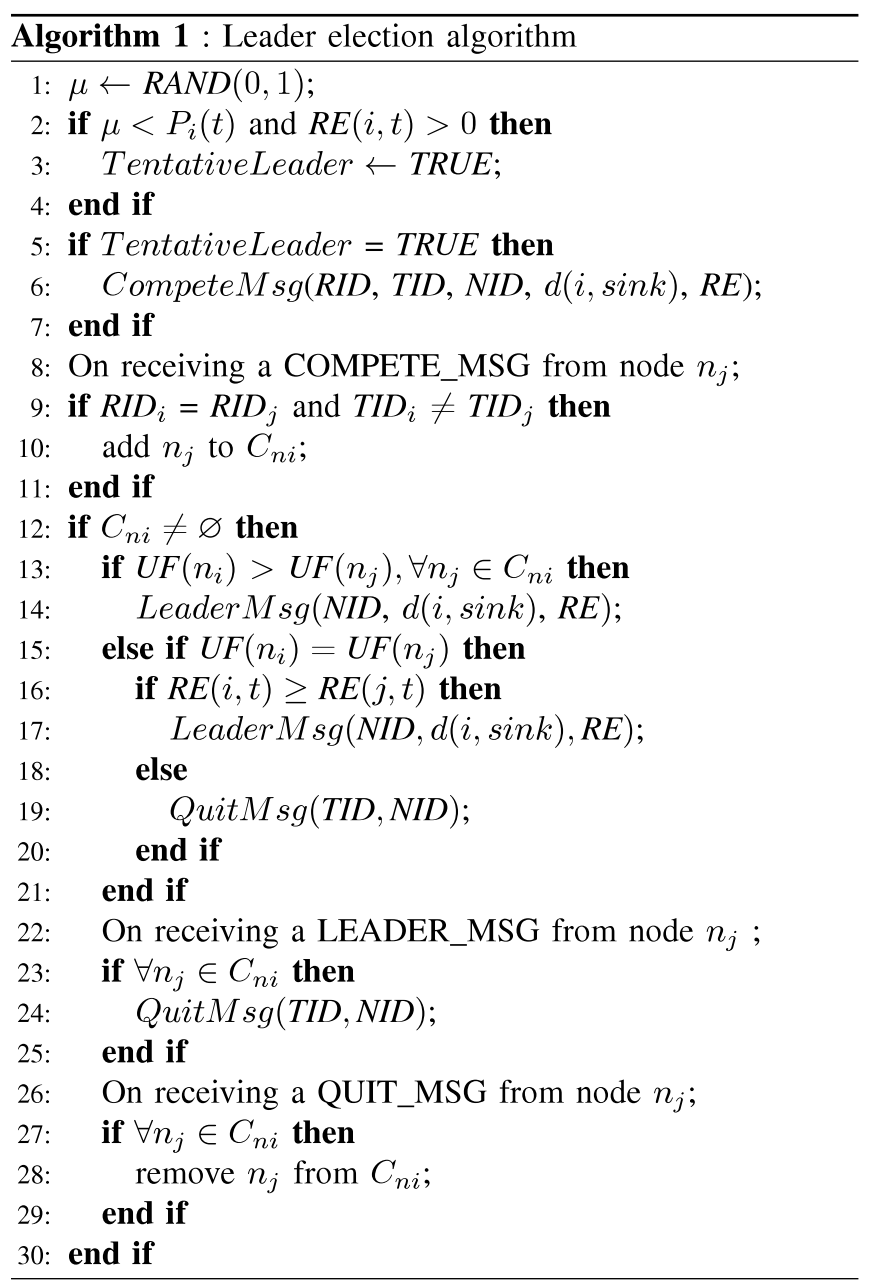

\section{B. Algorithm analysis}

According to Algorithm 1, the leader election process is message driven, thus we discuss its complexity below.

Lemma 1. The control overhead complexity of the leader election algorithm is $O(I)$, where $I$ is the number of nodes.

Proof: Observing EELE, every node sends out a few quite short control messages each round without iteration. At the start of the leader election phase, $\sum_{k=1}^{K} g_{k} P_{i}(t)=K$ tentative leaders are produced and each of them broadcasts a COMPETE_MSG. As described aforementioned, $P_{i}(t)$ determines the number of tentative leaders. Then, each of them either broadcasts a LEADER_MSG to act as a final leader or broadcasts a QUIT_MSG to be a normal node. For only one leader is elected in one region, they send out one LEADER_MSG and $(K-1)$ QUIT_MSGs. Once the leader has been 
elected, the normal nodes will decide their communication mode and inform the leader by a short control message. Thus, the messages add up to $2 K+2(I-1)$ per round. Since $K<I$, the total asymptotic order of the control overhead is $O(I)$.

Remark 1. The number of nodes in a WBAN is extremely limited by nature of the network. Consequently, Lemma 1 verifies the message overhead of Algorithm 1 is very small.

\section{The EELE MeChANISM}

A fundamental problem in WBANs is to maximize the network lifetime under given energy constraints. In order to conserve the energy and ensure that all nodes die approximately at the same time, the operation of EELE is divided into rounds to make the nodes in different regions wake up alternately as shown in Fig. 1. After the initial phase, each round begins with the set-up phase when the leader is elected, followed by the scheduling phase. At last, the network accesses into the steady-state phase when the nodes transmit packets to the sink once a communication mode is chosen. The time line is shown in Fig. 1. The following sub-sections describe the operation procedure of EELE in details.

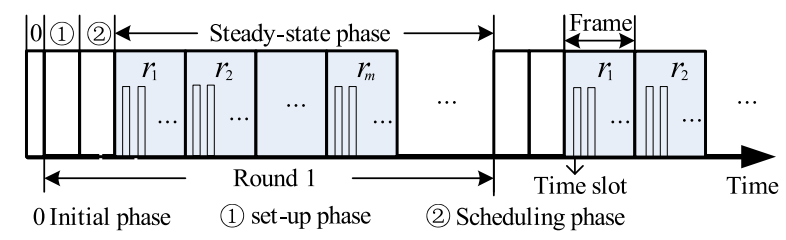

Fig. 1. The time line of EELE operation.

\section{A. Initial phase}

Initially, the deployment is established using recommendation of medical institutes and some optimal deployment methods [10]. Then, the sink broadcasts a "hello" message including its location to all nodes. After receiving the message, each node can compute and store the approximate distance to the sink based on the received signal. Subsequently, each node establishes an information table which consists of its RID, TID, $N I D, R E$ and its distance to the sink. At the end of each round, the nodes update their residual energy immediately.

\section{B. Set-up phase}

In this phase, the main task is to elect the leader in each region. All nodes are initialized with an equal amount of energy, and they always send the data about the physiological parameters during the active period. According to the algorithm 1 in section II, the tentative leaders will compete for the final leader. Simultaneously, the other nodes turn into sleep state. Once the leaders have been eventually settled, they must inform the sink and all the normal nodes of their roles as the leaders for the current round. Meanwhile, the sleeping nodes must wake up and receive the final message LEADER_MSG. Each normal node determines its communication mode in this round and informs the leader by sending a short message. Then the leader aggregates the messages from all normal nodes into one single packet, and transfers it to the sink.

\section{Scheduling phase}

After receiving the aggregative messages from the $M$ leaders, the sink sets up a TDMA schedule and transmits it to each leader. On receiving the TDMA schedule, each leader sets up a sub-TDMA schedule and informs its members. Through the sub-TDMA schedule, the leader manages only one node for each type to work while the others to get into sleep status and ensure that every active normal node just transmits once per round. Subsequently, the steady-state operation can begin.

\section{Steady-state phase}

The steady-state phase is divided into frames which are assigned to the different regions based on the TDMA schedule. And each frame is further partitioned into time-slots which are assigned to nodes based on the sub-TDMA schedule. Finally, each node sends its data to the sink or the leader during its assigned time-slot, respectively.

The operation flowchart of the aforementioned EELE mechanism on a node is shown in Fig. 2.

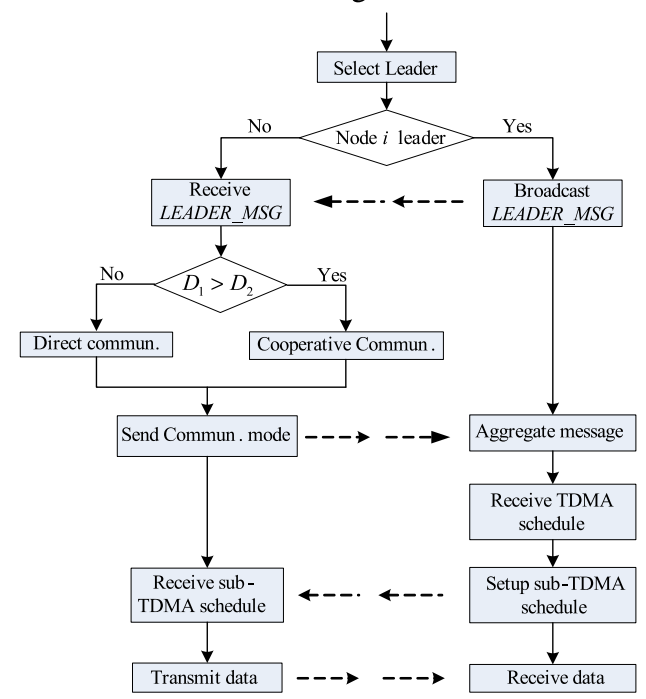

Fig. 2. The flowchart of EELE mechanism.

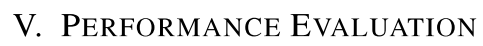

In this section, we evaluate the performance of the proposed EELE mechanism and compare it with two other schemes derived from LEACH [6] and HEED [8] which are the most classical energy-efficient mechanisms for WSNs: LEACHAnalogous (LEACH-A) and HEED-Analogous (HEED-A). Unless otherwise specified, an ideal MAC layer and error-free communication links are assumed.

\section{A. Performance metrics}

In the simulation, we assess the performance of EELE in terms of:

- Network lifetime: Lifetime is the vital criterion for evaluating the performance of WBANs, which is defined as the time until the first node or the last node dies;

- Energy characteristics: To verify the effectiveness and efficiency of EELE on the energy conservation, the average residual energy and the energy efficiency will be 
evaluated. The definition of the later is the ratio of the total received data to the total energy consumption.

- Throughput: Throughput is defined as the average receiving data successfully per round.

\section{B. Simulation settings}

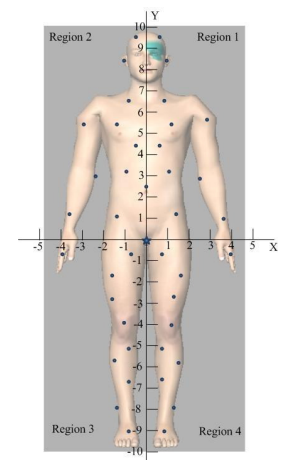

Fig. 3. Sensor location

According to the symmetry of human body and the location of the sink node, a WBAN is partitioned into 4 equal regions as shown in Fig. 3. Due to nature of the network, the number of nodes in a WBAN is limited in the range of 20-50 [1]. Consequently, 10 sensor nodes are deployed in each region and categorized into 5 types based on their monitoring function, separately. And the detailed simulation parameters are given in Table 1, where the radio parameters of the Nordic nRF2401 transceiver [10] are employed. Moreover, every simulation results are the average of 100 independent experiments.

TABLE I

Simulation SETTINGS

\begin{tabular}{|c|c|}
\hline Parameter & Setting \\
\hline One region coverage & $(0,0) \sim(50,100)$ \\
\hline Initial energy & $0.1 \mathrm{~J}$ \\
\hline$E_{\text {Txelec }}, E_{\text {Rxelec }}$ & $16.7 \mathrm{~nJ} / \mathrm{bit}, 36.1 \mathrm{~nJ} / \mathrm{bit}$ \\
\hline$\epsilon_{a m p}(n)$ & $1.97 \mathrm{~nJ} /\left(\right.$ bit $\left.\cdot \mathrm{m}^{n}\right),(n=3.11)$ \\
\hline$E_{D A}$ & $5 \mathrm{~nJ} / \mathrm{bit} /$ signal \\
\hline Data packet size & 4,000 bits \\
\hline
\end{tabular}

\section{Simulation results and analysis}

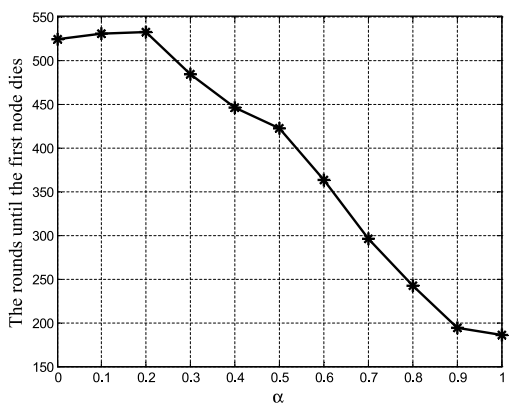

Fig. 4. The impact of $\alpha$ on the network lifetime in EELE.

1) Network lifetime: In EELE mechanism, we choose the leaders through the maximum utility function with the residual energy and the location of the node taken into consideration. As described in section III, $\alpha$ determines the proportional factor of the residual energy and the distance, which means the preference for the distance or the residual energy when electing the leader. Thus we need adopt an optimal value to prolong the network lifetime.

The relation between $\alpha$ and the network lifetime is shown in figure 4 . When $\alpha$ varies from 0 to 1 , the influence of the residual energy on the utility function decreases gradually, while that of the distance increases. Particularly, when $\alpha=0$, it implies the node with the most residual energy is elected as the leader. And when $\alpha=1$, it means that the node closest to the sink will be the leader. Obviously, there is an optimal value of $\alpha=0.2$ when the network can achieve the maximum lifetime. This result indicates the energy is more prioritized when electing the leader. Therefore, we use $\alpha=0.2$ in the following simulations.

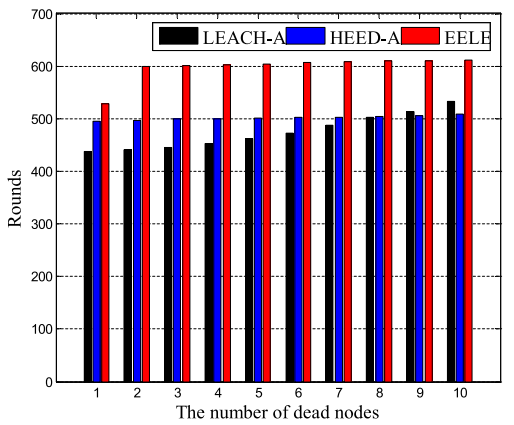

Fig. 5. The time until a given number of nodes die.

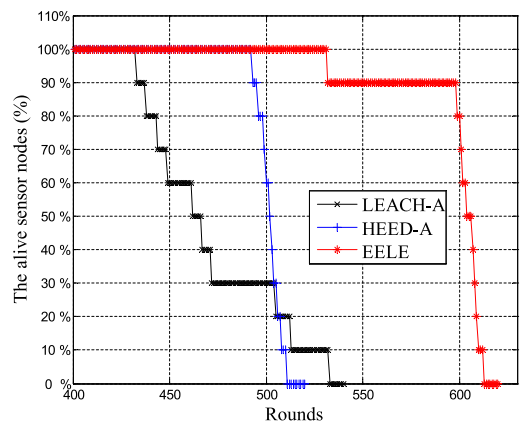

Fig. 6. The percent of the alive nodes over time.

In Fig. 5 and 6, we assess the network lifetime in terms of the dead nodes and the percentage of remaining alive nodes. As shown in Fig. 5, EELE mechanism clearly improves network lifetime over LEACH-A and HEED-A. Furthermore, the Fig. 6 shows the total number of nodes that remain alive over the simulation time. In EELE there are quite more alive nodes than those in others at the same time. Specifically, the first node died at the 529th round in EELE which are 34 and 92 rounds later than that in HEED-A and that in LEACH-A, respectively. Moreover, there are still $90 \%$ nodes alive in EELE when all nodes are dead in both HEED-A and LEACH-A.

The reason is that every node has the equal probability to become the leader in LEACH-A. Once the node with rather less residual energy is elected as the leader, it will be dead easily and fast. While in HEED-A the far nodes have a bigger probability to become leaders, resulting in the more energy dissipated. But this is avoided in EELE since the far node will be a leader if and only if its residual energy is considerably larger than that of the near one, and this obviously reduces 
the probability of the far node to become the leader. Even if the far node competes as the leader, the closer node can communicate directly with the sink in EELE, which alleviates the burden of the leader. The above results shows EELE can indeed efficiently prolong the network lifetime.

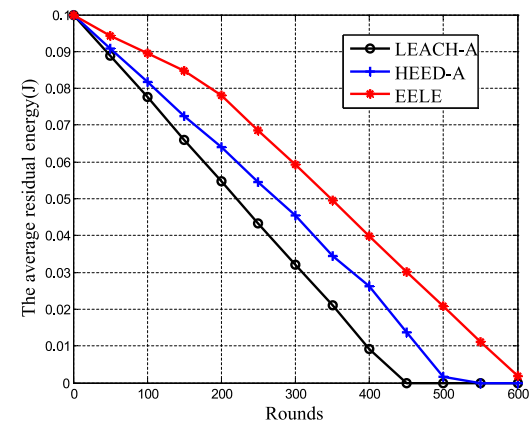

Fig. 7. The average residual energy over time.

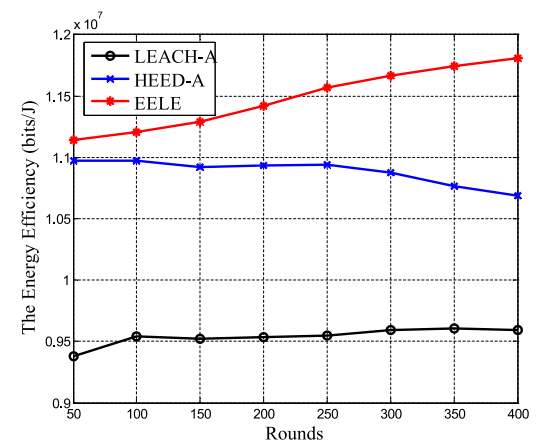

Fig. 8. The network energy efficiency over time.

2) Energy characteristic: In this part, we investigate the average residual energy per node and the energy efficiency. Fifty rounds of simulations are sampled and the results are shown in Fig. 7 and Fig. 8. EELE achieves more residual energy and higher energy efficiency than the others.

In LEACH-A and HEED-A, all the packets of normal nodes must be sent to the leader and then the leader transfers them to the sink. But a far node can compete as the leader with the same probability as a closer node in LEACH-A. As also, a far one competes as the leader with a increasing probability as the simulation runs in HEED-A. Once the far one wins as the leader, the closer ones must transmit their packets to it. As a result, the leader has to consume more energy to transfer the same data over a longer distance.

Differently, EELE scheme lowers the probability of a far node to be the leader unless it has obviously much more residual energy. Moreover, the adopted hybrid communication mode benefits to relieve the burden of the far nodes and the leaders. Accordingly, the energy efficiency of EELE increases slightly as the operation of the WBAN. Therefore, the energy efficiency of EELE is much better than that of LEACH-A and HEED-A.

3) Throughput: The Fig. 9 shows that the EELE is better than LEACH-A and HEED-A in the term of the throughput as the initial energy increases. Accurately, EELE outperforms LEACH-A and HEED-A over up to $51.7 \%$ and $33.7 \%$, respectively.It is the superiority of EELE mechanism in the effectiveness and energy efficiency on energy conservation that contributes to more delivered packets.

According to the above analysis, we can draw a conclusion that EELE is more suitable for the energy constraint and smallscale network such as WBANs.

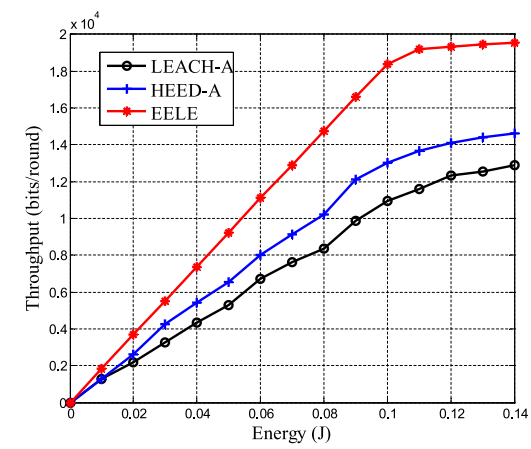

Fig. 9. The network throughput over different initial energy.

\section{CONCLUSiON}

In this paper, a novel energy efficient leader election mechanism for prolonging the network lifetime is presented in WBANs. Firstly, a hybrid communication mode was developed such that the burden of the leader and the far node was relieved. Secondly, we designed a distributed leader election algorithm which comprehensively considers both the residual energy and the location of the node. The theoretical analysis proved the overhead of the algorithm was small. Subsequently, the operation of EELE is introduced in de details. Finally, simulation results showed that EELE mechanism could reduce the energy consumption, prolong the network lifetime and increase the network throughput effectively.

\section{REFERENCES}

[1] Latré B, Braem B, Moerman I, et al., "A survey on wireless body area networks," Wireless Networks, vol. 17, pp. 1-18, Jan. 2011.

[2] Movassaghi S, Abolhasan M, Lipman J, et al., "Wireless Body Area Networks: A Survey," Communication Surveys \& Tutorials, IEEE, PP. 1-29, Jan. 2014.

[3] Ullah S, Higgins H, Braem B, et al., "A comprehensive survey of wireless body area networks," Journal of medical systems, vol. 36, pp. 1065-1094, June, 2012.

[4] Cavallari R, Martelli F, Rosini R, et al., "A Survey on Wireless Body Area Networks: Technologies and Design Challenges," Communication Surveys \& Tutorials, IEEE, PP.1-23, Feb. 2014.

[5] Wei D, Jin Y, Vural S, et al., "An energy-efficient clustering solution for wireless sensor networks," IEEE Transactions on Wireless Communications, vol.10, pp. 3973-3983,2011.

[6] Heinzelman W B, Chandrakasan A P, Balakrishnan H, "An applicationspecific protocol architecture for wireless microsensor networks," IEEE Transactions on Wireless Communications, vol.1, pp. 660-670, 2002.

[7] Randriatsiferana R S A, Lorion R, Alicalapa F, et al., " Energy-efficient clustering algorithm based on energy variance for Wireless Sensor Networks," IEEE International Conference on Smart Communications in Network Technologies (SaCoNeT), June, 2013, pp. 1-5.

[8] Younis O, Fahmy S, "HEED: a hybrid, energy-efficient, distributed clustering approach for ad hoc sensor networks," IEEE Transactions on Mobile Computing, vol. 3, pp. 366-379, Oct. 2004.

[9] Kumar D, "Performance analysis of energy efficient clustering protocols for maximizing lifetime of wireless sensor networks," IET Wireless Sensor Systems, vol.4, pp.9-16, March,2013.

[10] E. Reusens, et al., Characterization of On-Body Communication Channel and Energy Efficient Topology Design for Wireless Body Area Networks, IEEE Transactions on Information Technology on Biomedecine,vol,13,pp.933-945, Nov. 2009. 